\title{
Mathematical modelling of concentration profiles for species transport through the single and the interconnected multiple-compartment systems
}

\author{
O. Adedire*, J. N. Ndam \\ Department of Mathematics, University of Jos, Nigeria.
}

\begin{abstract}
In this research work, we investigate the concentration profiles in the single and the interconnected multiple-compartment systems with sieve partitions for the transport of chemical species with second order chemical reaction kinetics. With assumption of unidirectional transport of chemical species and constant physical properties with same equilibrium contant, the developed partial differential equations representing the two systems are spatially discretised using the Method of Lines (MOL) technique and the resulting semi-discrete system of ODEs are solved using MATLAB ode15s solver. The results show that the interconnected multiple-compartment system has lower concentration profile than the single-compartment system for different values of diffusivities.
\end{abstract}

Keywords: Concentration profile, species, single-compartment, interconnected, multiple-compartment

Article History :

Received: 17 March 2020

Received in revised form: 02 May 2020

Accepted for publication: 04 May 2020

Published: 14 May 2020

(c)2020 Journal of the Nigerian Society of Physical Sciences. All rights reserved. Communicated by: T. Latunde

\section{Introduction}

Chemical reactor engineers have certain desired concentration profiles for optimum yield of products. While high concentration profile is preferable for some chemical species to yield adequate product, low concentration profile is desired for some other chemical species. The type of chemical reactor used is a factor that may determine the concentration profiles in such systems due to pressure, heat and possible consumption of the chemical species as a result of the presence of other reactions. Different chemical species have different diffusivities in the medium through which they are being transported. The medium through which a chemical species is being transported could be solid, liquid or gas medium.

${ }^{*}$ Corresponding author tel. no:

Email address: dharenss@gmail .com (O. Adedire )
In literature, researchers have modelled high and low concentration profiles for different diffusion values in different media. Gaiseanu presented analysis on diffusion of boron in silicon at high surface concentration beginning from some diffusion constants and his conclusion is centred on the dependence of diffusion coefficient on concentration for some given parameters whose details can be found in his work [1]. Chepurniy and Savage also investigated the effect of diffusion on concentration profile in a solar pond and concluded that constant value of diffusivity existed in the medium considered [2]. While investigating probe effects on concentration profiles in the diffusion layer, Critelli et al. [3] solved a pure diffusion problem for a onecomponent two dimensional axisymmetric model. They concluded that positioning of a probe like a microelectrode close to a working electrode interferes with local potential and concentration distributions. 
Yang and Lue examined coupled concentration-dependent diffusivities of ethanol and water mixture through a polymeric membrane. While determining effects on pervaporative flux and diffusivities profiles, they concluded that the concentration profiles exhibited a linear to concave response as a function of depth in the considered membranous medium [4]. There have been various works on concentration profiles and diffusivities of chemical species in different media [5-11].

Zhou et al. [12] generated complex concentration profile by partial diffusive mixing in multi-stream laminar flow. One of the key insights in their work is that details in the concentration profile can be tuned with geometrical and operating parameters. Adedire and Ndam investigated model of chlorine decay through water and intermediate Pseudomonas aeruginosa in multiple-compartment isothermal system [13]; they however did not compare their work with single compartment system. Researchers in literature have not compared the concentration profiles between the single and the interconnected multiplecompartment systems for the transport of chemical species with second order chemical reaction kinetics.

The research question is to determine whether single compartment system will have higher concentration profile than interconnected multiple-compartment system for chemical species with second order chemical reaction kinetics as time progressively increases. Would there be higher concentration profile for chemical species with higher diffusivities than for those with lower diffusivities?

In this study, we intend to compare the concentration profiles in both the single-compartment system and the interconnected multiple-compartment system for the transport of chemical species with second order chemical reaction kinetics with respect to diffusivities. The interconnected multiple-compartment system is such that each compartment is separated by sieve partitions allowing transport of chemical species through each compartment of the system.

The subsequent part of this paper is organised as follows: section 2 deals with model development. Section 3 deals with numerical simulation of the model equations. While in section 4 , results and discussion from the perspective of the considered systems will be addressed, conclusion comes up in section 5 .

\section{Model Development}

The development of the model in this work is centred on treating fluid as a continuum [14]. Let any chemical species be a substance distributed in $\mathbb{R}^{n}$ with boundary $\partial \Omega$ and let $\hat{n}$ be an outward normal to $\partial \Omega$ and define a function

$\Psi_{\varphi}(\bar{x}, t): \mathbb{R}^{n} \times \mathbb{R} \rightarrow \mathbb{R}$

where $\Psi_{\varphi}(\bar{x}, t)$ is the concentration of any chemical species $\varphi$ in some units of measurements having $\bar{x} \in \mathbb{R}^{n}$ with $\bar{x}=x_{1}, x_{2}, \ldots x_{n}$ at any time t. Let $q \in \mathbb{N}$ be total number of compartments and $\gamma \in\{1,2,3,4,5\}$ be compartment $1,2,3,4$ and 5 respectively. For $q=1, \gamma=1, n=1, x_{1}=x$ we set up mass balance equations assuming that convection and diffusion are taking place along the $x$-axis as shown in Figure 1. For chemical species $\varphi$ with flux $q_{\varphi}$ and reaction term $R_{\varphi}$ over infinitesimal thickness $\Delta x$ in $x$-direction of $\gamma^{\text {th }}$-compartment of the reactor, the mass balance gives

$$
\begin{aligned}
& \left.(\Delta x) \Psi_{\varphi}(x, t)\right|_{t}-\left.(\Delta x) \Psi_{\varphi}(x, t)\right|_{t+\Delta t} \\
& =\left.v \Psi_{\varphi}(x, t)\right|_{x} \Delta t-\left.v \Psi_{\varphi}(x, t)\right|_{x+\Delta x} \Delta t \\
& +\left.q_{h_{1} x}\right|_{x} \Delta t-\left.q_{h_{1} x}\right|_{x+\Delta x} \Delta t+R_{\varphi} \Delta x \Delta t
\end{aligned}
$$

Division of (2) by $(\Delta x)(\Delta t)$ gives

$$
\begin{aligned}
\frac{\left.\Psi_{\varphi}(x, t)\right|_{t}-\left.\Psi_{\varphi}(x, t)\right|_{t+\Delta t}}{\Delta t}=-v \frac{\left.\Psi_{\varphi}(x, t)\right|_{x}-\left.\Psi_{\varphi}(x, t)\right|_{x+\Delta x}}{\Delta x} & \\
& -\frac{\left.q_{h_{1}}\right|_{x}-\left.q_{h_{1}}\right|_{x+\Delta x}}{\Delta x}+R_{\varphi}
\end{aligned}
$$

Take the limit of (3) as $\Delta x \rightarrow 0, \Delta t \rightarrow 0$ to get

$\frac{\partial \Psi_{\varphi}(x, t)}{\partial t}=-v_{1} \frac{\partial \Psi_{\varphi}}{\partial x}-\frac{\partial q_{\varphi}}{\partial x}+R_{\varphi}$

Assuming that the rate of consumption of the chemical species follows second order chemical reaction kinetics and choosing the flux $q_{\varphi}$ to follow Fick's law of diffusion [15], we have

$$
\begin{aligned}
& \frac{\partial \Psi_{\varphi}(x, t)}{\partial t}=-v \frac{\partial \Psi_{\varphi}(x, t)}{\partial x}+\frac{\partial}{\partial x}\left[D \frac{\partial\left[\Psi_{\varphi}(x, t)\right.}{\partial x}\right], \\
& -k_{\varphi}\left[\Psi_{\varphi}(x, t)\right]^{2} x_{L 0} \leqslant x \leqslant x_{\mu}, \mathrm{t} \geqslant 0
\end{aligned}
$$

with initial and boundary conditions given as

$\Psi_{\mathrm{fl} \varphi}(x, 0)=\Psi_{\mathrm{fl} \varphi 0}(x)$

$\Psi_{\mathrm{fl} \varphi}\left(x=x_{L 0}, t\right)=\Psi_{\mathrm{fl} \varphi}(t)$

$\Psi_{\mathrm{fl} \varphi}\left(x=x_{\mu}, t\right)=\Psi_{\mathrm{fl} \varphi E}\left(x=x_{\mu}, t\right)$

Following the same procedure used to obtain equations (5), (6), (7) and (8), we consider the following Figure 2 . For the parameters $q=5 ; \gamma=1,2,3,4,5 ; n=1, x_{1}=x$, we obtain the following system of partial differential equations with assumption that convection and diffusion take place along $x$-axis as

$$
\begin{aligned}
& \frac{\partial \Psi_{1 \varphi}(x, t)}{\partial t}=-v \frac{\partial \Psi_{1 \varphi}(x, t)}{\partial x}+\frac{\partial}{\partial x}\left[D_{1} \frac{\partial \Psi_{1 \varphi}(x, t)}{\partial x}\right] \\
& -k_{\varphi}\left[\Psi_{1 \varphi}(x, t)\right]^{2}, x_{L} \leqslant x \leqslant x_{1}, \mathrm{t} \geqslant 0 \\
& \frac{\partial \Psi_{2 \varphi}(x, t)}{\partial t}=-v \frac{\partial \Psi_{2 \varphi}(x, t)}{\partial x}+\frac{\partial}{\partial x}\left[D_{2} \frac{\partial \Psi_{2 \varphi}(x, t)}{\partial x}\right] \\
& -k_{\varphi}\left[\Psi_{2 \varphi}(x, t)\right]^{2}, x_{1} \leq x \leq x_{2}, \mathrm{t} \geq 0
\end{aligned}
$$

$$
\begin{aligned}
& \frac{\partial \Psi_{3 \varphi}(x, t)}{\partial t}=-v \frac{\partial \Psi_{3 \varphi}(x, t)}{\partial x}+\frac{\partial}{\partial x}\left[D_{3} \frac{\partial \Psi_{3 \varphi}(x, t)}{\partial x}\right] \\
& -k_{\varphi}\left[\Psi_{3 \varphi}(x, t)\right]^{2}, x_{2} \leq x \leq x_{3}, \mathrm{t} \geq 0
\end{aligned}
$$

$$
\frac{\partial \Psi_{4 \varphi}(x, t)}{\partial t}=-v \frac{\partial \Psi_{4 \varphi}(x, t)}{\partial x}+\frac{\partial}{\partial x}\left[D_{4} \frac{\partial \Psi_{4 \varphi}(x, t)}{\partial x}\right]
$$




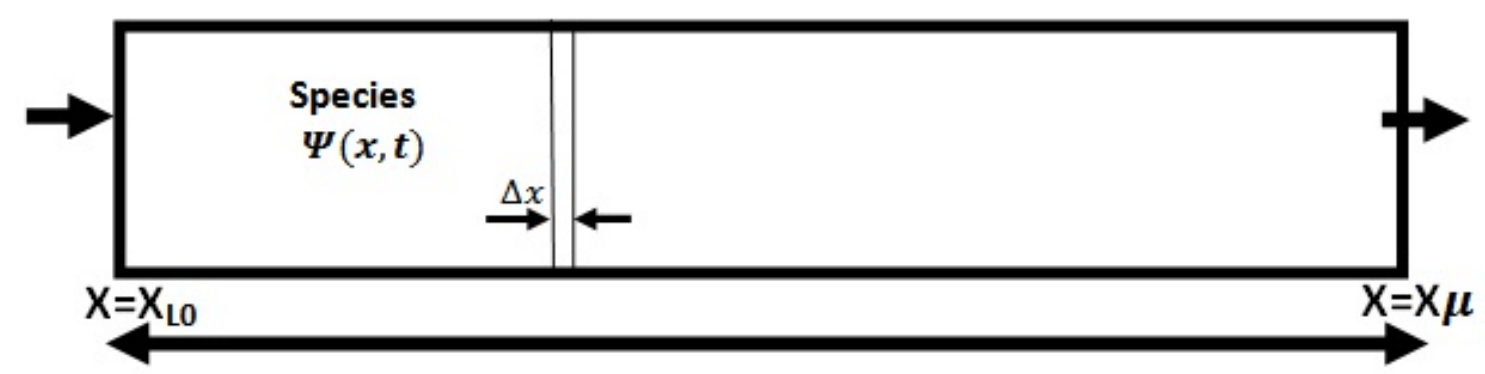

Figure 1: Schematic representation of species transport through $(\mathrm{q}=1)$ single-compartment chemical reactor system.

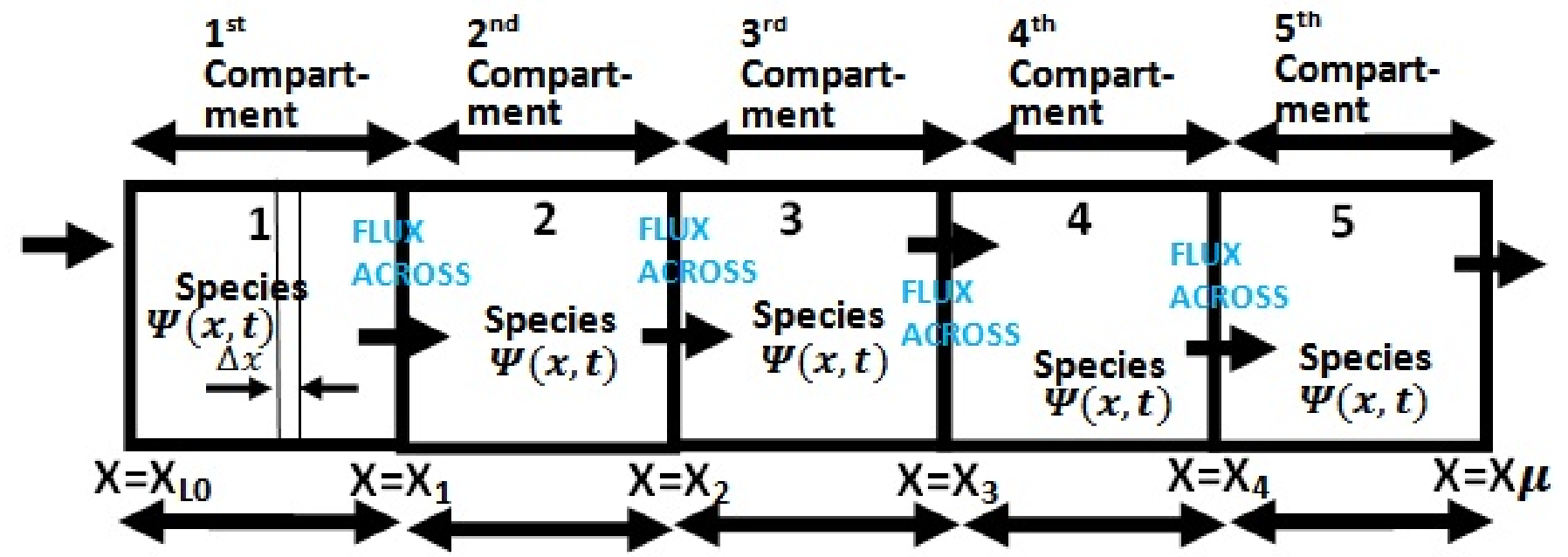

Figure 2: Schematic representation of species transport through interconnected (q=5)- multiple-compartment chemical reactor system.

$$
\begin{aligned}
& -k_{\varphi}\left[\Psi_{4 \varphi}(x, t)\right]^{2}, x_{3} \leq x \leq x_{4}, \mathrm{t} \geq 0 \\
& \frac{\partial \Psi_{5 \varphi}(x, t)}{\partial t}=-v \frac{\partial \Psi_{5 \varphi}(x, t)}{\partial x}+\frac{\partial}{\partial x}\left[D_{5} \frac{\partial \Psi_{5 \varphi}(x, t)}{\partial x}\right] \\
& -k_{\varphi}\left[\Psi_{5 \varphi}(x, t)\right]^{2}, x_{4} \leqslant x \leqslant x_{\mu}, \mathrm{t} \geqslant 0
\end{aligned}
$$

Equation (9) has initial and boundary conditions

$\Psi_{1 \varphi}(x, 0)=\Psi_{1 \varphi 0}(x)$

$\Psi_{1 \varphi}\left(x=x_{L 0}, t\right)=\Psi_{1 \varphi}(t)$

$\Psi_{1 \varphi}\left(x=x_{1}, t\right)=k\left[\Psi_{2 \varphi}\left(x=x_{1}, t\right)\right]$

Equations (10), (11) and (12) for $\gamma=2,3$ and 4 have the initial and boundary conditions

$\Psi_{\gamma \varphi}(x, 0)=\Psi_{\gamma \varphi 0}(x)$

$\frac{\partial \Psi_{\mathrm{fl} \varphi}\left(x=x_{\gamma}, t\right)}{\partial x}=\frac{D_{(\gamma-1) \varphi}}{D_{\gamma \varphi}} \frac{\partial \Psi_{(\gamma-1) \varphi}\left(x=x_{(\gamma-1)}, t\right)}{\partial x}$

$\Psi_{\gamma \varphi}\left(x=x_{\gamma}, t\right)=k\left[\Psi_{(\gamma+1) \varphi}\left(x=x_{\gamma}, t\right)\right]$

Equation (13) for $\gamma=5$ has the initial and boundary conditions given as

$\Psi_{5 \varphi}(x, 0)=\Psi_{5 \varphi 0}(x)$ $\frac{\partial \Psi_{5 \varphi}\left(x=x_{4}, t\right)}{\partial x}=\frac{D_{4 \varphi}}{D_{5 \varphi}} \frac{\partial \Psi_{4 \varphi}\left(x=x_{4}, t\right)}{\partial x}$

$\Psi_{5 \varphi}\left(x=x_{5}, t\right)=\Psi_{5 \varphi 0}(t)$

While the Neumann boundary conditions (18) and (21) show continuity of flux of species $\varphi$ from $\gamma^{\text {th }}$ compartment to the adjacent $(\gamma+1)^{\text {th }}$ compartment, the Dirichlet boundary conditions (16), (19) and (22) show the link between the concentrations in one compartment with the concentration in the preceding compartment in a way showing relationship at the boundaries.

\subsection{Existence and Uniqueness of Solution}

The solutions of the governing equations (5)-(8) and (9)-(22) which are PDEs and their auxiliary conditions exist and are unique; hence they are well-posed system of PDEs [16]. Detailed proofs of the existence and uniqueness of the governing model equations leading to their well-posedness will not be considered here to avoid repetition as they have been extensively proved elsewhere. For further analysis and detailed proofs, the reader is referred to [16] and to [17] and references contained therein.

\section{Numerical Simulation}

The governing model equation (5) together with its auxiliary conditions (6)-(8) for $(\mathrm{q}=1)$ single-compartment system 
and the system of equations (9)-(13) together with their auxiliary conditions (14)-(22) for interconnected multiple-compartment system are solved using Method of Lines (MOL) technique. We performed numerical simulation after only the spatial derivatives of the governing PDEs have been discretised with finite difference approximations. To this end, let $x=x_{i}$ such that $i \in \mathbb{N}$ be an index representing positions on grids in $x$. We consider first order approximations to $\frac{\partial \Psi}{\partial x}$ for the convective part of the PDEs as

$\frac{\partial \Psi(x, t)}{\partial x} \approx \frac{\Psi_{i}-\Psi_{i-1}}{\Delta x}+O(\Delta x)$

Also, for the diffusive part, second order finite difference approximations to the second order derivative $\frac{\partial^{2} \Psi}{\partial x^{2}}$ are considered as

$\frac{\partial^{2} \Psi(x, t)}{\partial x^{2}} \approx \frac{\Psi_{i+1}-2 \Psi_{i}+\Psi_{i-1}}{\Delta x^{2}}+O\left(\Delta x^{2}\right)$

The terms $O(\Delta x)$ and $O\left(\Delta x^{2}\right)$ are the truncation error of approximation of finite difference scheme obtained from Taylor series. The number of points on the grids in $x$ is chosen as $\mathrm{M}$ such that at the first boundary (left-end) of $x$ a value of $i=1$ is assigned and at the last boundary (right-end) of $\mathrm{x}$ a value of $\mathrm{i}=\mathrm{M}$ is chosen.

For $q=1, \gamma=1, n=1$ substitution of (23) and (24) into (5) results in

$$
\begin{gathered}
\frac{d \Psi(t)_{(\varphi)}}{d t}=-v \frac{\Psi(t)_{(\varphi)} i \Psi(t)_{(\varphi)} i-1}{\Delta x}+D\left[\frac{\Psi(t)_{(\varphi)} i+1-2 \Psi(t)_{(\varphi)}+\Psi(t)_{(\varphi)} i-1}{\Delta x^{2}}\right] \\
-k_{\varphi}\left[\Psi(t)_{(\varphi)}\right]^{2}, \quad 1 \leqslant i \leqslant M
\end{gathered}
$$

and (6), (7) and (8) are semi-discretised as

$\Psi(t=0)_{(\varphi) i}=\Psi_{\varphi 0}(x(i))$

$\Psi(t)_{(\varphi) 1}=\Psi_{\varphi}(t)$

$\Psi(t)_{(\varphi) \mathrm{M}}=\Psi(t)_{(\varphi) 0 \mathrm{M}}$

For $q=5 ; \gamma=1,2,3,4,5$, substitution of (23) and (24) into (9), (10), (11), (12) and (13) results in

$$
\begin{aligned}
& \frac{d \Psi(t)_{(1 \varphi) i}}{d t}=-v_{1} \frac{\Psi(t)_{(1 \varphi) i}-\Psi(t)_{(1 \varphi) i-1}}{\Delta x} \\
& +D_{1}\left[\frac{\Psi(t)_{(1 \varphi) i+1}-2 \Psi(t)_{(1 \varphi) i}+\Psi(t)_{(1 \varphi) i-1}}{\Delta x^{2}}\right] \\
& -k_{1 \varphi}\left[\Psi(t)_{(1 \varphi) i}\right]^{2}, \quad 1 \leqslant i \leqslant M_{(1)} \\
& \frac{d \Psi(t)_{(2 \varphi) i}}{d t}=-v_{2} \frac{\Psi(t)_{(2 \varphi) i}-\Psi(t)_{(2 \varphi) i-1}}{\Delta x} \\
& +D_{2}\left[\frac{\Psi(t)_{(2 \varphi) i+1}-2 \Psi(t)_{(2 \varphi) i}+\Psi(t)_{(2 \varphi) i-1}}{\Delta x^{2}}\right] \\
& -k_{2 \varphi}\left[\Psi(t)_{(2 \varphi) i}\right]^{2}, \quad 1 \leqslant i \leqslant M_{(2)} \\
& \frac{d \Psi(t)_{(3 \varphi) i}}{d t}=-v_{3} \frac{\Psi(t)_{(3 \varphi) i}-\Psi(t)_{(3 \varphi) i-1}}{\Delta x}
\end{aligned}
$$

$$
\begin{aligned}
& +D_{3}\left[\frac{\Psi(t)_{(3 \varphi) i+1}-2 \Psi(t)_{(3 \varphi) i}+\Psi(t)_{(3 \varphi) i-1}}{\Delta x^{2}}\right] \\
& -k_{3 \varphi}\left[\Psi(t)_{(3 \varphi) i}\right]^{2}, \quad 1 \leqslant i \leqslant M_{(3)} \\
& \frac{d \Psi(t)_{(4 \varphi) i}}{d t}=-v_{4} \frac{\Psi(t)_{(4 \varphi) i}-\Psi(t)_{(4 \varphi) i-1}}{\Delta x} \\
& +D_{4}\left[\frac{\Psi(t)_{(4 \varphi) i+1}-2 \Psi(t)_{(4 \varphi) i}+\Psi(t)_{(4 \varphi) i-1}}{\Delta x^{2}}\right] \\
& -k_{4 \varphi}\left[\Psi(t)_{(4 \varphi) i}\right]^{2}, \quad 1 \leqslant i \leqslant M_{(4)} \\
& \frac{d \Psi(t)_{(5 \varphi) i}}{d t}=-v_{5} \frac{\Psi(t)_{(5 \varphi) i}-\Psi(t)_{(5 \varphi) i-1}}{\Delta x} \\
& +D_{5}\left[\frac{\Psi(t)_{(5 \varphi) i+1}-2 \Psi(t)_{(5 \varphi) i}+\Psi(t)_{(5 \varphi) i-1}}{\Delta x^{2}}\right] \\
& -k_{5 \varphi}\left[\Psi(t)_{(5 \varphi) i}\right]^{2}, \quad 1 \leqslant i \leqslant M_{(5)}
\end{aligned}
$$

where $M_{(1)}, M_{(2)}, M_{(3)}, M_{(4)}, M_{(5)}$ are the number of points on the grid in $x$ in each compartment $\gamma=1,2,3,4,5$. The initial and boundary conditions (14),(15) and (16) are semi-discretised as

$\Psi(t=0)_{(1 \varphi) i}=\Psi_{1 \varphi 0}(x(i))$

$\Psi(t)_{(1 \varphi) 1}=\Psi_{1 \varphi}(t)$

$\Psi(t)_{(1 \varphi) \mathrm{M}_{(1)}}=k \Psi(t)_{(2 \varphi)} \mathrm{M}_{(1)}$

and equations (17), (18) and (19) are semi-discretised for (30), (31) and (32) as

$$
\begin{aligned}
\Psi(t= & 0)_{(\gamma \varphi) i}=\Psi_{\gamma \varphi 0}(x(i)) \\
& \frac{\Psi(t)_{(\gamma \varphi) \mathrm{M}_{(\gamma-1)}}-\Psi(t)_{(\gamma \varphi) \mathrm{M}_{(\gamma-1)}-1}}{\Delta x} \\
& =\frac{D_{(\gamma-1) \varphi}}{D_{\gamma \varphi}} \frac{\Psi(t)_{[(\gamma-1) \varphi] \mathrm{M}_{(\gamma-1)}}-\Psi(t)_{[(\gamma-1) \varphi] \mathrm{M}_{(\gamma-1)}-1}}{\Delta x}
\end{aligned}
$$$$
\Psi(t)_{(\gamma \varphi) \mathrm{M}_{(\gamma)}}=k \Psi(t)_{[(\gamma+1) \varphi]} \mathrm{M}_{(\gamma)}
$$

Finally, equations (20),(21) and (22) are semi-discretised as

$$
\begin{gathered}
\Psi(t=0)_{(5 \varphi) i}=\Psi_{5 \varphi 0}(x(i)) \\
\frac{\Psi(t)_{(5 \varphi)} \mathrm{M}_{(4)}-\Psi(t)_{(5 \varphi) \mathrm{M}_{(4)}-1}}{\Delta x} \\
=\frac{D_{(4) \varphi}}{D_{5 \varphi}} \frac{\Psi(t)_{4 \varphi \mathrm{M}_{(4)}}-\Psi(t)_{4 \varphi \mathrm{M}_{(4)}-1}}{\Delta x} \\
\Psi(t)_{(5 \varphi) \mathrm{M}_{(5)}}=k \Psi(t)_{5 \varphi 0 \mathrm{M}_{(5)}}
\end{gathered}
$$




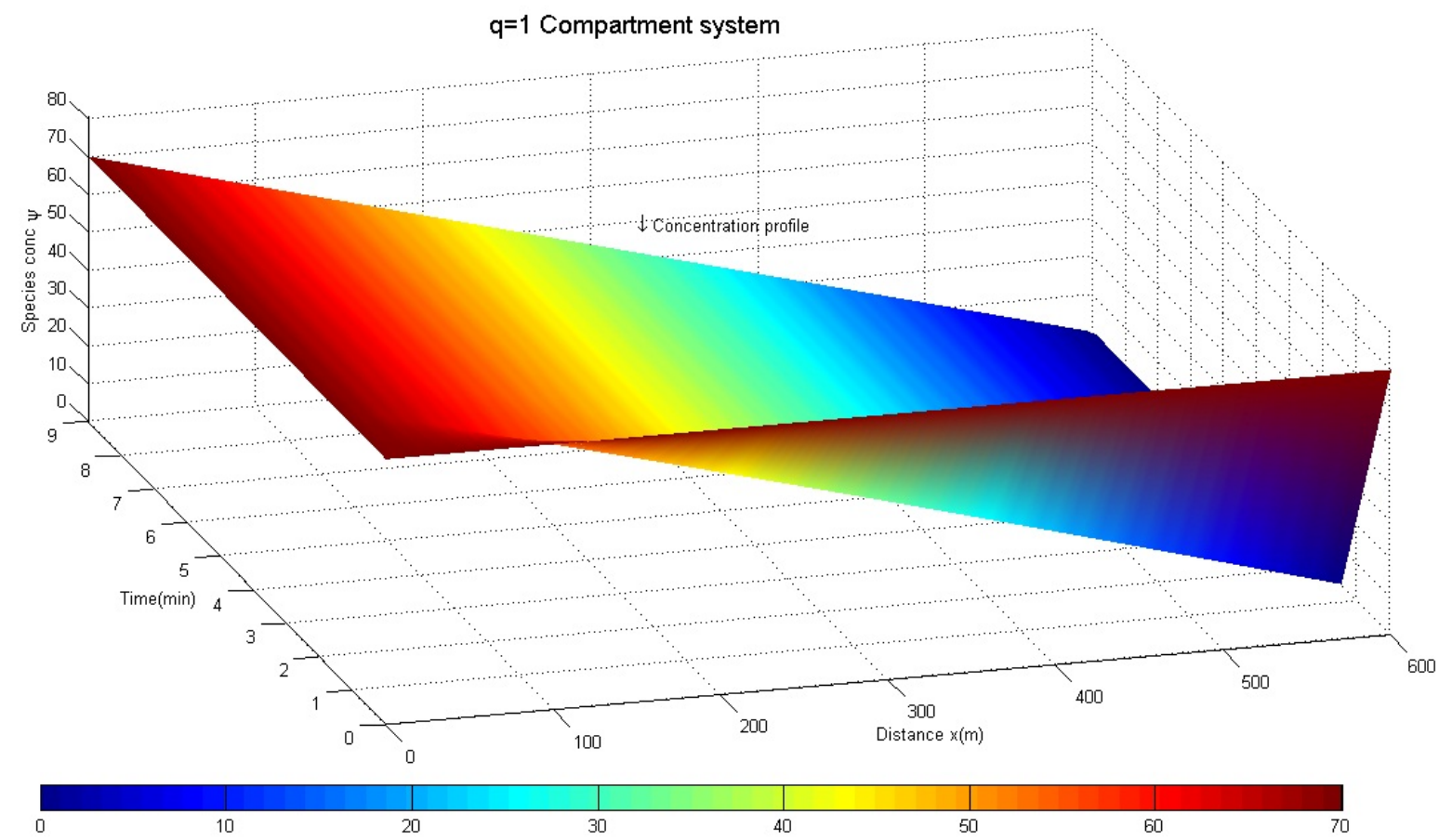

Figure 3: Species concentration profile for diffusivity $5.4 \times 10^{1} \mathrm{~m}^{2} / \min \gamma=1(\mathrm{q}=1)$ single-compartment system at time $\mathrm{t}=9$ minutes.
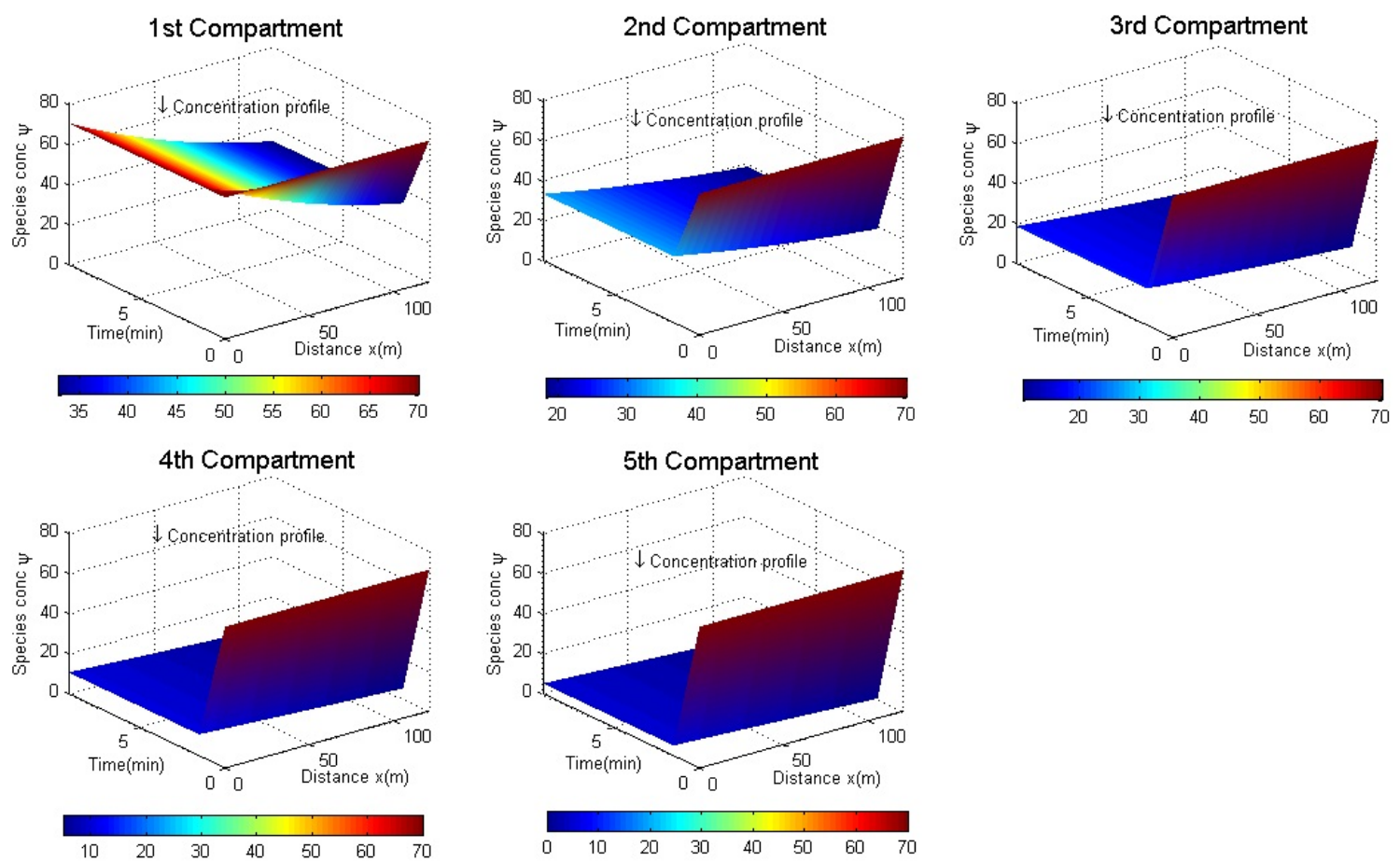

Figure 4: Species concentration profile for diffusivity $5.4 \times 10^{1} \mathrm{~m}^{2} / \min \gamma=1,2,3,4,5$ of interconnected $(\mathrm{q}=5)$ multiple-compartment system at time $\mathrm{t}=9$ minutes.

\section{Results and Discussion}

For parameters $q=1, \gamma=1, n=1$, the total length of the system is $600 \mathrm{~m}$ with a constant boundary value $\psi_{\varphi 0}=70 \mathrm{mg} / \mathrm{L}$. The second order reaction rate constant $k_{\varphi}$ is $1.2 \times 10^{1} \mathrm{~L} \mathrm{mg}$ $\mathrm{min}^{-1}$, the diffusivities $D_{\varphi}$ are taken to be $5.4 \times 10^{1} \mathrm{~m}^{2} / \mathrm{min}$ and $5.4 \times 10^{2} \mathrm{~m}^{2} / \mathrm{min}$ respectively with initial concentration of the chemical species taken as $\psi_{A \varphi 0}=70 \mathrm{mg} / \mathrm{L}$.

For parameters $q=5 ; \gamma=1,2,3,4,5 ; n=1$, the length of each compartment is $120 \mathrm{~m}$ so that the total length gives $600 \mathrm{~m}$. A constant boundary value $\psi_{1 \varphi 0}=70 \mathrm{mg} / \mathrm{L}$ is used, second order reaction rate constants $k_{i \varphi}(i=1,2,3,4,5)$ are taken to be $1.2 \times 10^{1} \mathrm{~L} \mathrm{mg} \mathrm{min}{ }^{-1}$ with diffusivities $D_{i \varphi}(i=1,2,3,4,5)$ also 
$q=1$ Compartment system

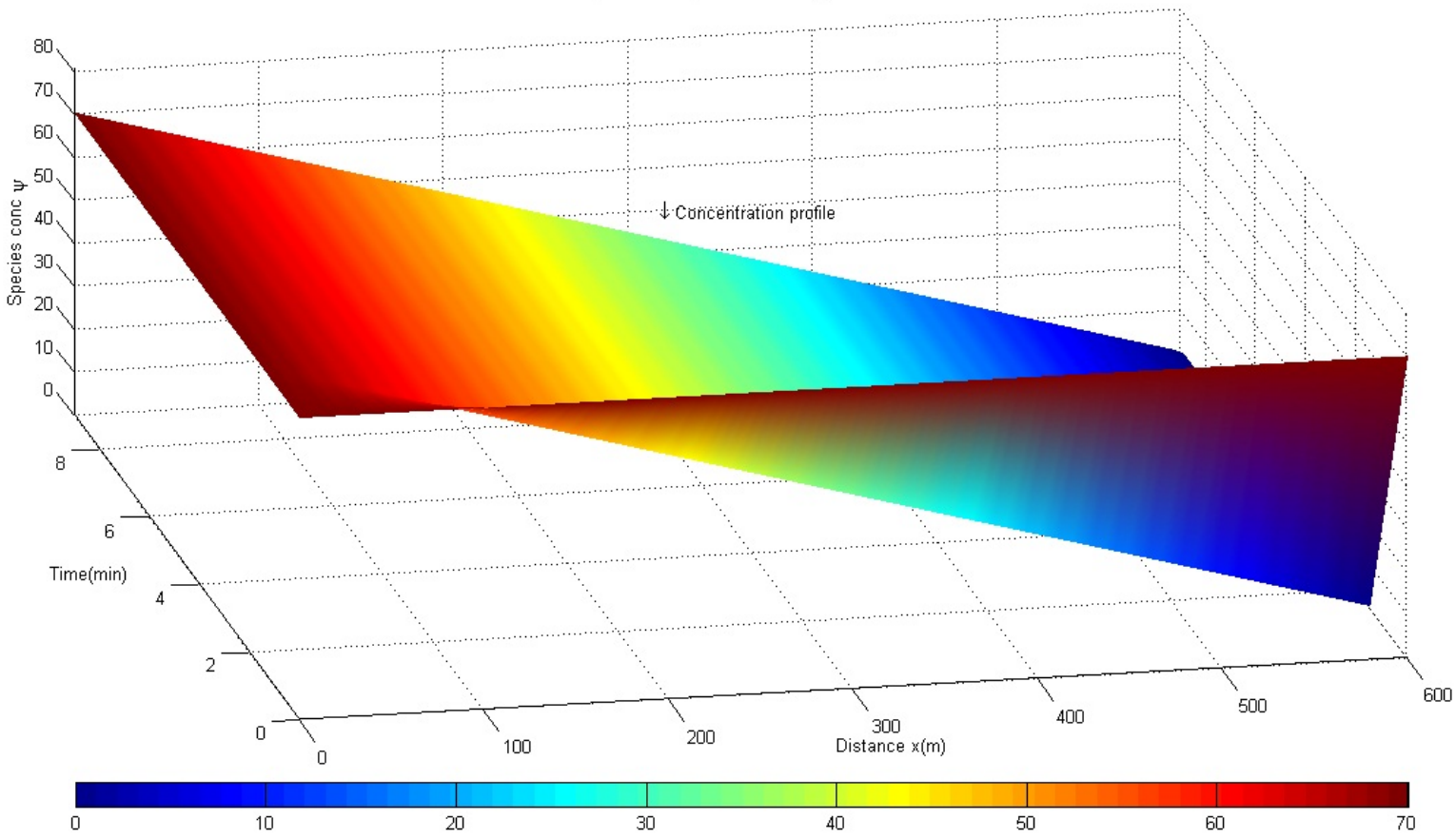

Figure 5: Species concentration profile for diffusivity $5.4 \times 10^{2} \mathrm{~m}^{2} / \min \gamma=1$ of $(\mathrm{q}=1)$ single-compartment system at time $\mathrm{t}=9$ minutes.

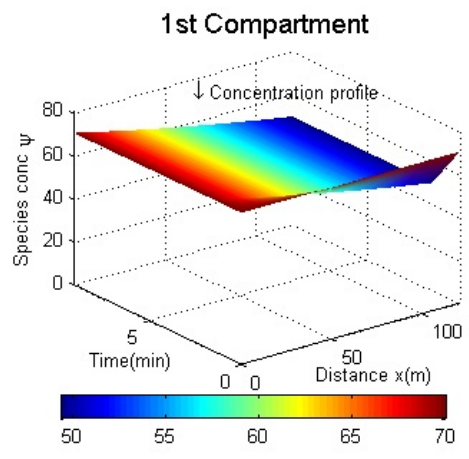

4th Compartment

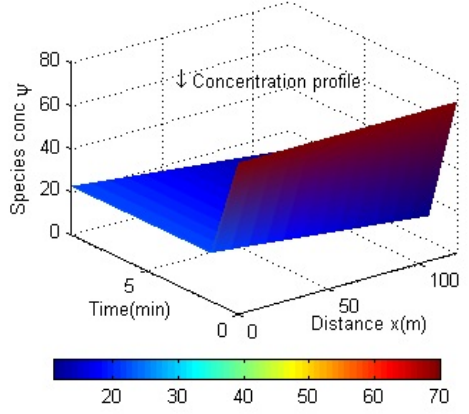

2nd Compartment

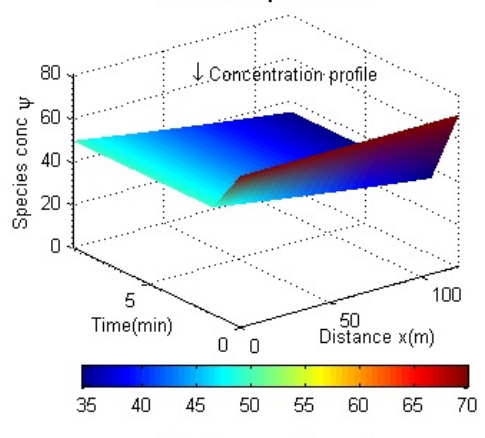

5th Compartment

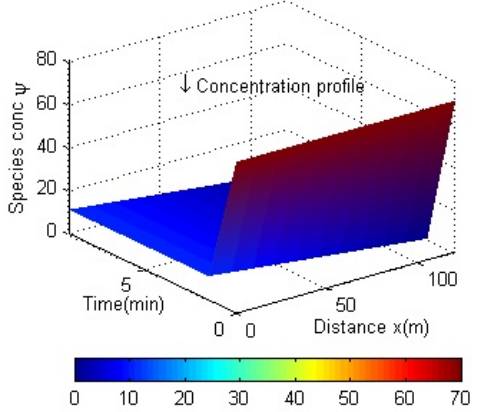

3rd Compartment

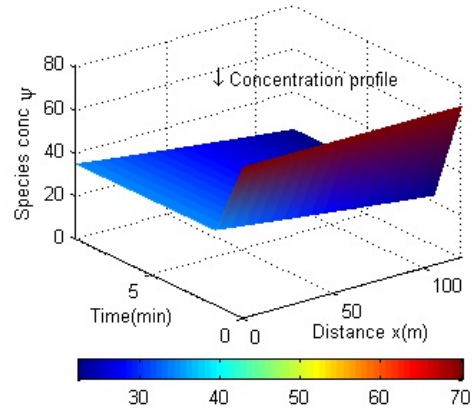

\section{0}


of handling such systems of ODEs with variable step size, the results are displayed for $\mathrm{t}=9$ minutes as shown in Figures 3 and 4.

Analogously, the same set of parameters used to obtain Figures 3 and 4 but with different diffusivities $D_{\varphi}$ and $D_{i \varphi}(i=$ $1,2,3,4,5)$ taken to be $5.4 \times 10^{2} \mathrm{~m}^{2} / \mathrm{min}$ are used and the results are displayed in Figures 5 and 6 at $t=9$ minutes for $(q=1)$ single-compartment system and interconnected $(q=5)$ multiplecompartment system respectively.

Simulation of the governing model equations for different values of diffusivities are used to investigate effects of diffusivity on the concentration profile of chemical species undergoing second order chemical reaction kinetics in each compartment of interconnected ( $\mathrm{q}=5$ )- multiple-compartment system. The results from Figure 4 show that for diffusivities $D_{i \varphi}(i=$ $1,2,3,4,5)$ given as $5.4 \times 10^{1} \mathrm{~m}^{2} / \mathrm{min}$ at $t=9$ minutes, the values of the concentration at the end of the boundary of each compartment of the system has the concentration values of 32.87 $\mathrm{mg} / \mathrm{L}, 18.19 \mathrm{mg} / \mathrm{L}, 10.49 \mathrm{mg} / \mathrm{L}, 4.99 \mathrm{mg} / \mathrm{L}$ and $0.00 \mathrm{mg} / \mathrm{L}$ respectively. On the other hand, for diffusivities $D_{i \varphi}(i=1,2,3,4,5)$ given as $5.4 \times 10^{2} \mathrm{~m}^{2} / \mathrm{min}$ measured at the same time $\mathrm{t}=9 \mathrm{~min}-$ utes, the values of concentration at the end of each compartment are obtained as $49.32 \mathrm{mg} / \mathrm{L}, 34.43 \mathrm{mg} / \mathrm{L}, 22.23 \mathrm{mg} / \mathrm{L}$, $11.01 \mathrm{mg} / \mathrm{L}$ and $0.00 \mathrm{mg} / \mathrm{L}$ for $\gamma=1,2,3,4,5$ respectively as shown in Figure 6. The mid-point of the multiple-compartment system of length $600 \mathrm{~m}$ is $300 \mathrm{~m}$, so that interconnected (q=5)compartment system has its mid-point in the third compartment at the point $x=60 \mathrm{~m}$ which is equivalent to $300 \mathrm{~m}$ of the entire system. While concentration of the species at $300 \mathrm{~m}$ of the system is $13.90 \mathrm{mg} / \mathrm{L}$ for diffusivity of $5.4 \times 10^{1} \mathrm{~m}^{2} / \mathrm{min}$, the concentration of the species is $28.13 \mathrm{mg} / \mathrm{L}$ for diffusivity of $5.4 \times 10^{2} \mathrm{~m}^{2} / \mathrm{min}$ at the mid-point of the system at $\mathrm{t}=9$ minutes.

From the results, there is higher concentration profile when diffusivities $D_{i \varphi}(i=1,2,3,4,5)$ are given as $5.4 \times 10^{2} \mathrm{~m}^{2} / \mathrm{min}$ than when diffusivities $D_{i \varphi}(i=1,2,3,4,5)$ are given as $5.4 \times 10^{1}$ $\mathrm{m}^{2} /$ min at $\mathrm{t}=9$ minutes. This means that chemical species with higher diffusivities in $(\mathrm{q}=5)$-compartment system have higher concentration profile than those with lower diffusivities. As time t progressively increases, the concentration profile of chemical species with higher diffusivity still shows higher values than the one with lower diffusivity in $(\mathrm{q}=5)$-compartment system.

We also made comparison between ( $\mathrm{q}=1$ )-compartment systems for different values of diffusivities in order to ascertain whether the behavior exhibited by ( $q=5)$-compartment is consistent with that of $(\mathrm{q}=1)$-compartment system. From the results shown in Figure 3 at $\mathrm{t}=9$ minutes, the concentration at the mid-point of the system is $34.88 \mathrm{mg} / \mathrm{L}$ when diffusivity is taken to be $5.4 \times 10^{1} \mathrm{~m}^{2} / \mathrm{min}$ and $34.99 \mathrm{mg} / \mathrm{L}$ when diffusivity is $5.4 \times 10^{2} \mathrm{~m}^{2} / \mathrm{min}$. From the comparison made between the $(\mathrm{q}=1)$-compartment system and the interconnected $(q=5)$-compartment system for different values of diffusivities, there is higher concentration profile in chemical species with higher diffusivity than with the one with lower diffusivity in each system. However, the concentration profile in interconnected $(\mathrm{q}=5)$ multiple-compartment system is lower than that of $(\mathrm{q}=1)$ single-compartment system.

\section{Conclusion}

In this paper, we compared the concentration profile between $(\mathrm{q}=1)$ - single-compartment and interconnected $(\mathrm{q}=5)$ multiple-compartment systems for a chemical species with second order chemical reaction kinetics.

From the results, the $(\mathrm{q}=5)$ - multiple-compartment system has lower concentration profile than the ( $\mathrm{q}=1)$ - single-compartment system for different values of diffusivities. We conclude that the multiple-compartment chemical reactor system be considered whenever lower concentration profile is desired for certain chemical reactions. However, choice of a single-compartment chemical reactor may be made when high concentration profile is required for certain chemical reactions.

\section{Acknowledgments}

We thank the referees for the positive enlightening comments and suggestions, which have greatly helped us in making improvements to this paper.

\section{References}

[1] F. Gaiseanu, "On the diffusion profile of boron in silicon at high concentrations", Journal of physical state solid 77 (1983) 51, doi: 10.1002/pssa.2210770164.

[2] N. Chepurniy \& S.B. Savage, "Effect of diffusion on concentration profiles in a solar pond", Journal of solar energy, 17 (1975) 203, doi: 10.1016/0038-092x(75)90061-4.

[3] R. A. J. Critelli, M. Bertotti \& R.M. Torresi, "Probe effects on concentration profiles in the diffusion layer: Computational modeling and near-surface pH measurements using microelectrodes", Journal of electrochemical acta 511 (2018) 203, doi: 10.1016/j.electacta.2018.09.157.

[4] T. H. Yang \& S.J. Lue, "Coupled concentration-dependent diffusivities of ethanol/water mixtures through a polymeric membrane: Effect on pervaporative flux and diffusivity profiles", Journal of Membrane Science 443 (2013) 1, doi: 10.1016/j.memsci.2013.05.002.

[5] B. Michl, J. Schön, W. Warta \& M.C. Schubert, "The impact of different diffusion temperature profiles on iron concentrations and carrier lifetimes in multicrystalline silicon wafers", IEEE Journal of photovoltaics 71(12) (2013) 1768, doi: 10.1109/jphotov.2012.2231726.

[6] R. Schulz, K. Yamamoto, A. Klossek, F. Rancan, A. Vogt, C. Schütte, E. Rühl \& R. R. Netz, "Modeling of Drug Diffusion Based on Concentration Profiles in Healthy and Damaged Human Skin", Biophysical Journal 117(5) (2019) 998, doi: 10.1016/j.bpj. 2019.07.027.

[7] W. Wang \& Y. Lo, "Profile estimation of high concentration Arsenic or Boron diffusion in Silicon", Journal of IEEE Transactions on Electron Devices, 30 (12) (2013) 1828, doi: 10.1109/t-ed.1983.21453.

[8] A. C. Lasaga, "Influence of diffusion coupling on diagenetic concentration profiles", American Journal of Science, 281 (1981) 553.

[9] U. Rothhaar \& H. Oechsner, "Determination of diffusion induced concentration profiles in Cr203 films on ceramic A12Oa by Auger sputter depth profiling", Fresenius Journal of Analytical Chemistry, 353 (1995) 533, doi: $10.1007 /$ BF00321316.

[10] A. C. Leute, "The influence of the ideal mixing entropy on concentration profiles and diffusion paths in ternary systems", American Journal of Science, 281 (2010) 553, doi: 10.1016/j.jpcs.2010.08.018.

[11] G. Peev \& M. Rouseva, "A model for the concentration profile of PxOy in the interwafer gas phase on phosphorus doping of silicon using a solid planar diffusion source", Journal of modelling simulation material Science Engineering, 2 (1994) 1143, doi: 10.1088/0965-0393/2/6/006.

[12] Y. Zhou, Y. Wang, T. Mukherjee \& Q. Lin, "Generation of complex concentration profiles by partial diffusive mixing in multi-stream laminar flow", Lab Chip, Royal Society of Chemistry, 9 (2009) 1439, doi: $10.1039 / \mathrm{b} 818485 \mathrm{~b}$. 
[13] O. Adedire \& J. N. Ndam, "Mathematical modeling of chlorine decay through water and intermediate pseudomonas aeruginosa in multiplecompartment isothermal reactor", Journal of Advanced Mathematical Models and Applications 4 (2019) 167.

[14] P. A. Thompson, "Compressible-fluid dynamics", McGraw-Hill, Inc, New York

[15] H. J. V. Tyrrell, "The origin and present status of Fick's diffusion law",
Journal of Chemical Education 41(7) (1964) 397.

[16] J. Strikwerda, "Initial boundary value problems for incomplete parabolic systems", Journal of Communications on Pure and Applied Mathematics 30 (1977) 797.

[17] H. Kreiss \& J. Lorenz, "Initial-boundary value problems and the NavierStokes Equation", Academic Press, Inc, San Diego. 